ORIGINAL

\title{
Double radiofrequency ablation is more extensive with a spherical zone shape compared to single ablation in a pig liver model
}

\author{
Atsuo Oshio', Katsuyoshi Tamaki', Ichiro Shimizu', Hiroshi Fukuno', Mari Urata', \\ Susumu Ito', and Nobuya Sano ${ }^{2}$ \\ 'Departments of Digestive and Cardiovascular Medicine, Institute of Health Biosciences, The Uni- \\ versity of Tokushima Graduate School, and 'Division of Pathology, Tokushima University Hospital, \\ Tokushima, Japan
}

\begin{abstract}
:
Objective : We compared the duration of ablation and the area of coagulation necrosis between a single ablation method (SAM) and a double ablation method (DAM) with a 'multitined expandable' electrode (LeVeen $2 \mathrm{~cm}$ ) for radiofrequency ablation (RFA) using pig liver.

Method : In the SAM group, ablation was completed after the first roll-off. In the DAM group, an additional ablation was performed to achieve a second roll-off. The comparison was made of the time required for roll-off and the extent of coagulation necrosis between the both groups. The Ellipticity index (EI) quantitatively describes the shape of the general RF ablation zone in the axial plane.

Results : There was no statistically significant difference in the interval until the first roll-off between both groups (SAM group : 100.7 \pm 24.7 seconds vs DAM group : 103.2 \pm 37.7 seconds, $P=0.43$ ). In the DAM group, the interval from the start of the additional ablation until the second roll-off was $154.0 \pm 86.9$ seconds, longer than the interval for the first roll-off $(P=0.023)$. The extent of coagulation necrosis was significantly more extensive in the DAM group (axial diameter, mean \pm SD, $26.2 \pm 2.8 \mathrm{~mm}) \times($ maximal diameter : $29.3 \pm 1.6 \mathrm{~mm}) \times$ (minimal diameter : $26.5 \pm 3.6 \mathrm{~mm})$ compared to the SAM group with $(23.0 \pm 3.3 \mathrm{~mm}) \times(23.7 \pm$ $3.1 \mathrm{~mm}) \times(20.0 \pm 2.5 \mathrm{~mm})$, respectively. Although there was no statistically significant difference in the EI between both groups, macroscopically, the shape of coagulation necrosis tended to be non-spherical in the SAM group and spherical in the DAM group.

Conclusions : The DAM with a 'multitined expandable' electrode was more extensive with a spherical zone shape compared to the SAM. J. Med. Invest. 54 : 28-34, February, 2007
\end{abstract}

Keywords : radiofrequency ablation, pig liver, ablation method

Received for publication August 1, 2006 ; accepted September $19,2006$.

Address correspondence and reprint requests to Katsuyoshi Tamaki, Department of Digestive and Cardiovascular Medicine, Institute of Health Biosciences, The University of Tokushima Graduate School, Kuramoto-cho, Tokushima 770-8503, Japan and Fax : +81-88-633-9235

\section{INTRODUCTION}

Percutaneous therapies for hepatocellular carcinoma include ethanol infusion therapy, microwave coagulation therapy, and radiofrequency ablation (RFA). Of these therapies, RFA is less invasive, and 
the incidence of complications is lower $(1,2)$. Therefore, world-wide, it is the most commonly performed technique(3-5). For success, it is important to achieve coagulation necrosis at a low frequency at the target site of the tumor. The use of RFA causes extensive coagulation necrosis immediately after a single coagulation session, which distinguishes it from other therapies. Furthermore, several studies have been reported that the use of RFA results in a higher survival rate(6)compared to ethanol infusion therapy, and that RFA reduces the frequency of treatment compared to microwave coagulation therapy (7). The prediction of coagulation necrosis is important because online monitoring e.g. ultrasound is unreliable. Therefore, when an accurate treatment can not be performed by RFA, a lack of a peritumoral margin may lead to residual tumor cells, or an increase in the incidence of complications. An RFA-related complications have been reported in a number of studies $(8,9)$. Considering these limitations, it is important to predict the extent of coagulation necrosis achieved by ablation before surgery, which may lead to a more effective and safer treatment. Several factors are involved in the extent of ablation. In a previous study, we found that, when a major blood vessel is present in the estimated area of RFA, blood flow-related cooling effects are enhanced, thus decreasing the extent of coagulation necrosis(10). Therefore, several ablations should be performed to achieve tumor necrosis at the margin, although there is little basic information on differences in the extent of coagulation necrosis at the site of ablation between the single and double ablation methods. In addition, the size and shape of the area of coagulation necrosis is dependent on the RFA device used. When a 'multitined expandable' electrode needle is employed, in general, a double ablation is recommended ; the electrode needle punctures the target site, and ablation is initiated while completely developing the needle. After the first roll-off, an additional ablation is performed to obtain a second rolloff. In order to obtain more precise information on ablation performance for effective tumor necrosis, we compared the size and the shape of the area of coagulation necrosis and the duration of ablation between a single ablation and a double ablation using pig liver specimens.

\section{MATERIALS AND METHODS}

\section{Animals}

Two five-month-old pigs (body weight : $58 \mathrm{~kg}$ and $60 \mathrm{~kg}$, respectively) were used in the study. For anesthesia, xylazine at $5 \mathrm{mg} / \mathrm{kg}$ and ketamine at $5 \mathrm{mg} /$ $\mathrm{kg}$ was intramuscularly injected. After intubation, inhalation of oxygen and nitrous oxide at a ratio of $1: 1$ were performed, and anesthesia was maintained with 1.3\% halothane. An electrode pad was applied to the flank, and a laparotomy was performed by a median abdominal incision. The abdominal cavity was exposed using a wound retractor. Using a Logic 400MD ultrasonograph (Yokogawa Medical System, Tokyo, Japan), these pigs were the randomization within each pig for each random site randomization between single ablation method (SAM) group and double ablation method (DAM) group, after confirming the absence of major blood vessels at the periphery. In each animal, ablation was performed at 6 points (total of 12 points). Immediately after the end of RFA, these pigs were euthanized with an overdose of a potassium chloride solution. The liver was extirpated to macroscopically observe and measure the extent of the ablation.

\section{RF application}

In this experiment, we used an RF3000 radiofrequency generator (Boston Scientific, Natick, MA, USA). This system consisted of a LeVeen monopolar electrode needle (Boston Scientific) and an external grounding pad. The LeVeen electrode needle involved an insulation cannula measuring $17 \mathrm{G}$ in diameter and $15 \mathrm{~cm}$ in length. The cannula contained 8 staple-shaped electrode arms developing at the target site. We performed the ablation with an electrode needle measuring $2 \mathrm{~cm}$ following the recommended protocol. In the SAM group, ablation was started at an output of $30 \mathrm{~W}$ while completely developing the electrode needle. After the first roll-off was obtained, the ablation was then completed. In the DAM group, ablation was similarly started at an output of $30 \mathrm{~W}$ while completely developing the electrode needle. After the first roll-off was obtained, the ablation was discontinued for 30 seconds, and an additional ablation was then performed at an output corresponding to 50\% of that at roll-off until the second roll-off was obtained. When the first roll-off did not occur within 5 minutes, a second ablation was re-started from $70 \%$ power output. 


\section{Measurements and shapes at the site of ablation}

We evaluated the shapes of the 12 ablation sites. The extent of coagulation necrosis was measured, using the white area at the center as the border of coagulation necrosis ; the liver was first cut along an axial plane, defined as a plane along the electrode axis. The axial diameter $(\mathrm{AD})$ is defined as the distance in $\mathrm{mm}$ between the proximal and the distal edge of coagulation necrosis, in the axis of the electrode. The minimal transverse diameter (TDmin) is defined as the minimal distance in $\mathrm{mm}$ between two opposite edges of coagulation necrosis in the transverse plane. The maximal transverse diameter (TDmax) is defined as the maximal distance in $\mathrm{mm}$ between two opposite edges of co- agulation necrosis in the transverse plane (Fig. 1) (11). The ellipticity index (EI) quantitatively describes the general shape of coagulation necrosis in the axial plane and is calculated as the ratio of axial diameter $(\mathrm{AD})$ and mean transverse diameter ((TDmin+TDmax) /2) : EI=2AD/(TDmin+TDmax). Provided that $\mathrm{T}$ min is close to Tmax, a ratio of $1.0 \pm 0.1$ roughly corresponds to a spherical coagulation necrosis ; a ratio $>1.1$ to an elliptical coagulation necrosis ; and a ratio $<0.9$ to a flattened sphere.

\section{Statistical analysis}

The results are expressed as the mean $\pm \mathrm{SD}$. The diameter of the ablation site was compared between the two groups using the Mann-Whitney U-test. $\mathrm{P}<0.05$ was regarded as significant.

\section{Transverse diameter}
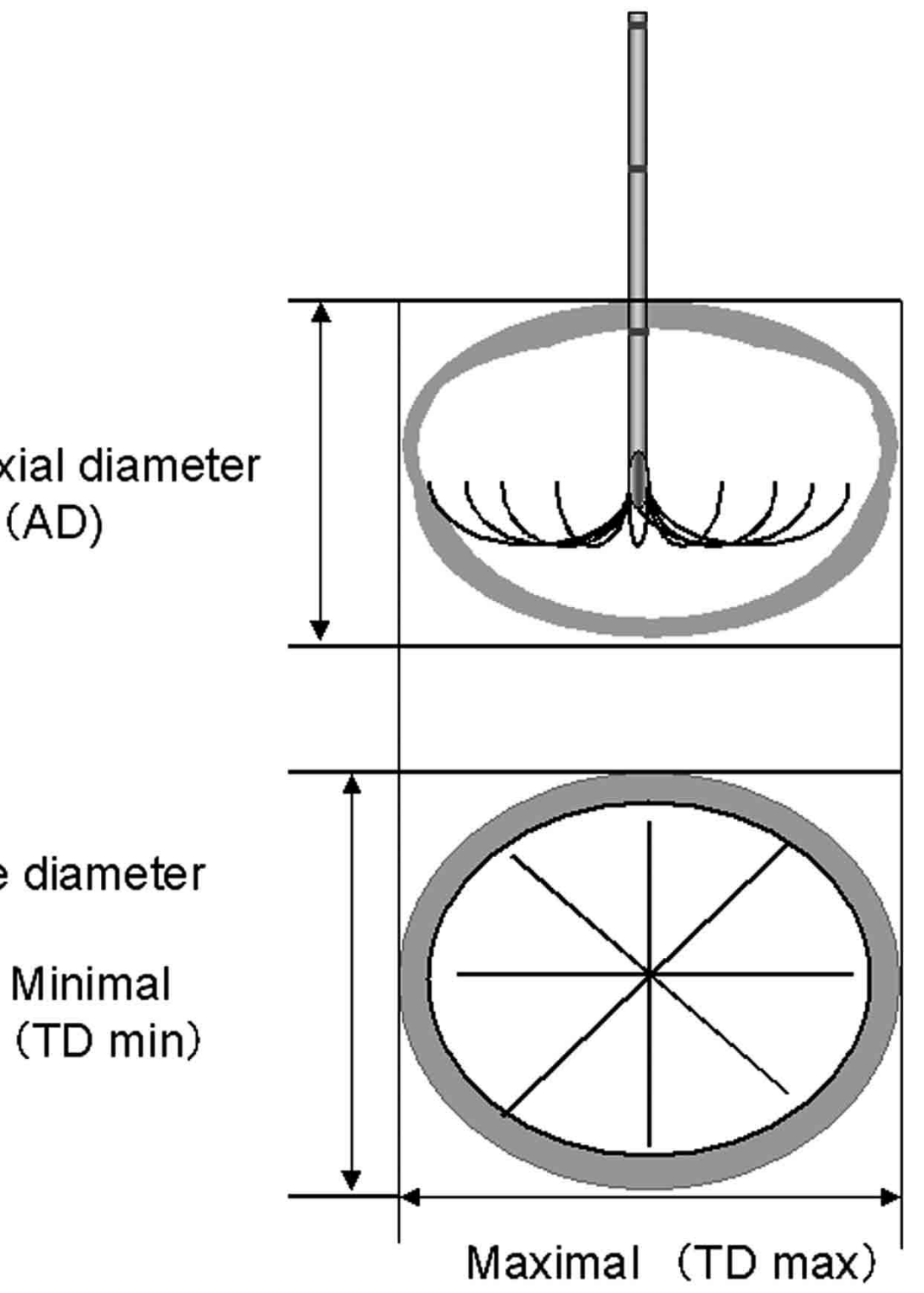

Fig. 1. Measurements at the site of ablation

$\mathrm{AD}$ : Axial diameter, TDmin : Minimal transverse diameter, TDmax : Maximal transverse diameter. 


\section{RESULTS}

Time required for roll-off during ablation

The interval until the first roll-off was $100.7 \pm$ 24.7 seconds in the SAM group and $103.2 \pm 37.7$ seconds in the DAM group. There was no significant difference between the two groups. There was also no significant difference in maximum output between the two groups.

In the DAM group (Table 1), the interval from the start of the additional ablation until the second roll-off was $154.0 \pm 86.9$ seconds, longer than the interval for the first roll-off. The first ablation required a higher output, although there was no significant difference in maximum output.

\section{Extent of coagulation necrosis}

As shown in Fig. 2, the extent of coagulation necrosis was $(\mathrm{AD}: 23.0 \pm 3.3) \times(\mathrm{TDmax}: 23.7 \pm 3.1) \times$
(TDmin : $20.0 \pm 2.5$ ) $\mathrm{mm}$ in the SAM group, and $(\mathrm{AD}: 26.2 \pm 2.8) \times(\mathrm{TDm}$ max : $29.3 \pm 1.6) \times(\mathrm{TDmin}:$ $26.5 \pm 3.6) \mathrm{mm}$ in the DAM group. The DAM group showed more extensive compared to the SAM with a significant difference.

\section{Lesion shape}

As shown in Fig. 2, there was no significant difference between the SAM group (EI : $1.08 \pm 0.27$ ) and the DAM group $(0.95 \pm 0.15)$. As shown in Table 2, in the SAM group, 1 of 6 coagulation necrosis was spherical (EI : 1.10) (Fig. 3A) ; 2 points were teardrop-shaped (EI : 0.75, 0.85) (Fig. 3B), and 3 points were ellipsoid in shape (EI:1.51, 1.15, 1, 14) (Fig. 3C).

In the DAM group, 4 of 6 ablation points were spherical (EI : $1.10,0.94,0.93,0.90) ; 1$ point was teardrop-shaped (EI : 0.70$) ; 1$ point was ellipsoid in shape (EI : 1.12)

Table 1. Comparison of ablation conditions between the single and double ablation groups

\begin{tabular}{lccc}
\hline & Single ablation & \multicolumn{2}{c}{ Double ablation } \\
\cline { 3 - 4 } & & 1st & 2nd \\
\hline Maximum output (W) & $40.0 \pm 6.3$ & $41.7 \pm 6.3$ & $22.5 \pm 6.9^{*}$ \\
\hline Interval until roll-off (seconds) & $100.7 \pm 24.7$ & $103.2 \pm 37.7$ & $154.0 \pm 86.9 * *$ \\
\hline
\end{tabular}

Values are means $\pm \mathrm{SD}(\mathrm{n}=6) .{ }^{*} \mathrm{P}=0.091$ compared to double ablation 1 st. $* * \mathrm{P}=0.023$ compared to double ablation 1 st.

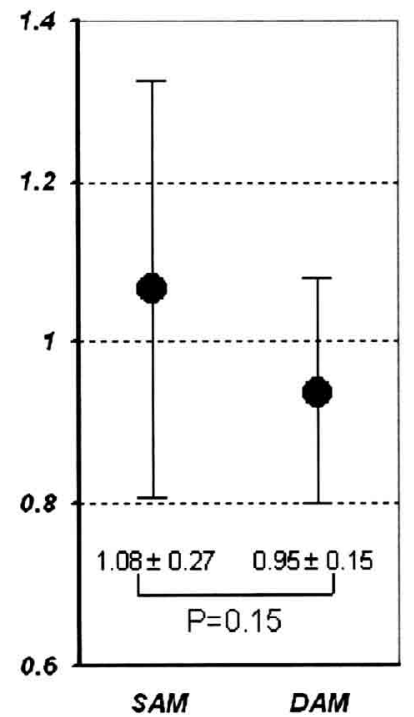

EI

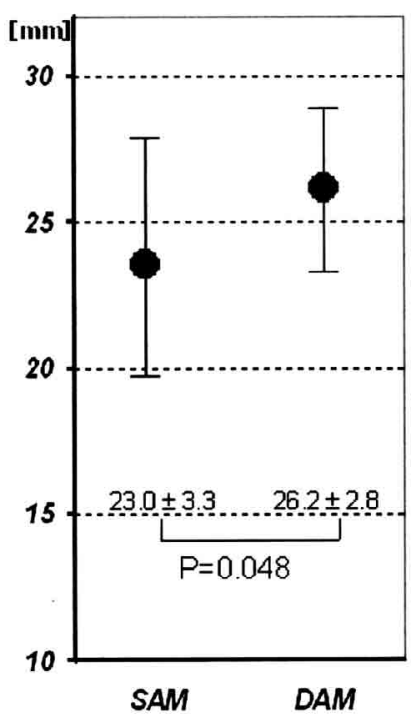

$A D$

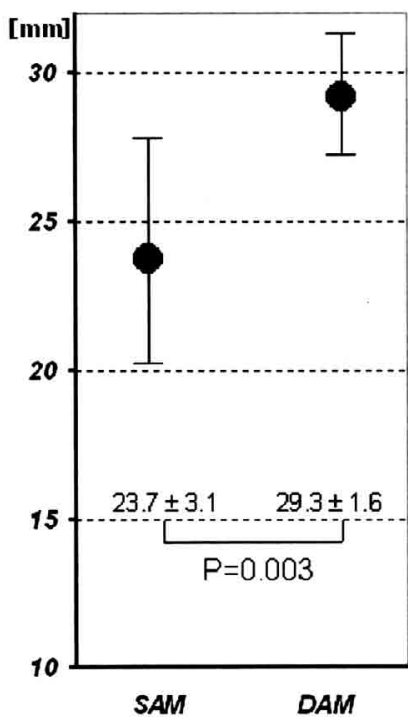

TDmax

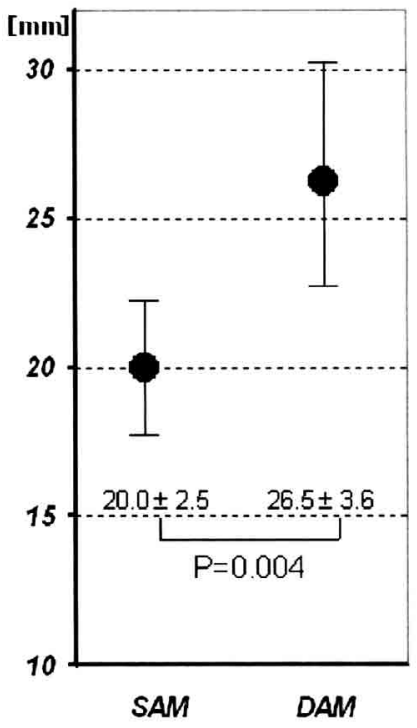

TDmin

Fig. 2. Comparison of the extent of ablation between the single and double ablation groups EI : Ellipticity index, SAM : Single ablation, DAM : Double ablation. Values are the means $\pm S D(n=6)$. 

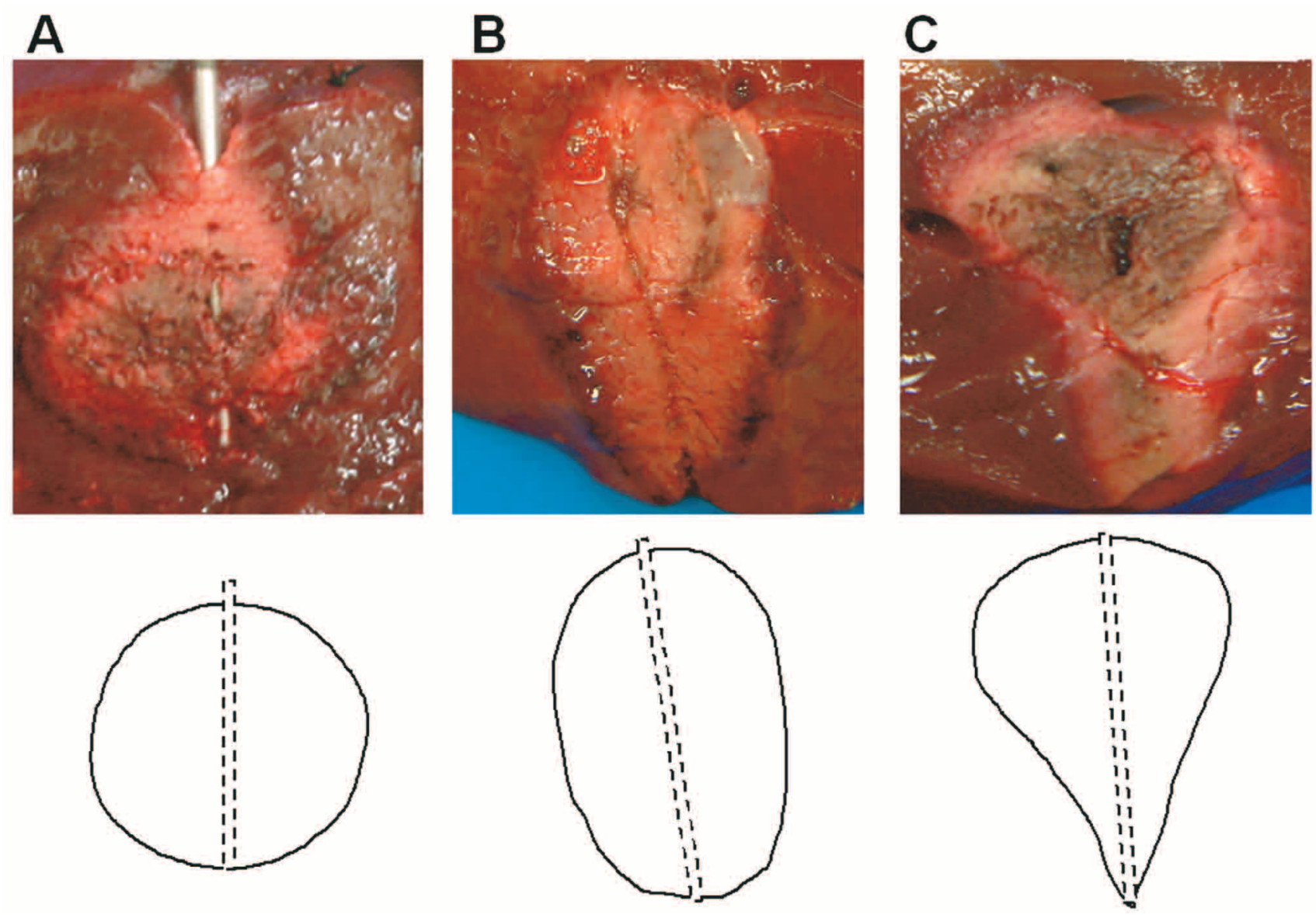

Fig. 3. Gross findings in resected livers after ablation

(A) Coagulation zone shapes are spherical, and the EI was $1.0 \pm 0.1$ (1 for the SAM group, 4 for the DAM group). (B)Coagulation zone shapes are elliptical, and the EI was more than 1.1 (3 for the SAM group, 1 for the DAM group). (C)Coagulation zone shapes are teardrop-shaped, and the EI was less than 0.9 (2 for the SAM group, 1 for the DAM group).

Table 2. Lesion shape of the single and double ablation groups

\begin{tabular}{cccc} 
& Spherical & Elliptical & Teardrop-shaped \\
\hline SAM & 1 & 3 & 2 \\
\hline DAM & 4 & 1 & 1
\end{tabular}

\section{DISCUSSION}

RFA is a widely used, therapeutic option for primary and secondary hepatic malignancies. When using the 'multitined expandable' electrode, it is nessecesary to achieve 'roll-off'. The 'roll-off' phenomenon represents a precipitous drop in power output coincident with a marked increase in tissue impedance due to coagulation necrosis.

Since most liver tumors are spherical, symmetrical ablation is important for reducing the risk of a regional relapse. For tumor ablation, the spherical area of coagulation necrosis must involve the entire tumor and the macroscopically normal tissue margin ; therefore, the size and the shape of coagulation necrosis may be important $(12,13)$. In the ablation protocol using an RF3000 device, a double ablation method is recommended by the manufactures, based on post experience ; after the first roll-off, an additional ablation is performed to obtain the second roll-off. $\mathrm{Ni}$, et al. reported that the double ablation method is more effective, due to the fact that current is stopped after the first ablation, not only because of irreversible charring, but also because of a reversible build-up of insulating gases, which disappear after a period of time(14). Therefore, in order to obtain more precise information on ablation performance for effective tumor necrosis, we compared the area of coagulation necrosis and the duration of ablation between a single ablation and a double ablation using pig liver specimens.

In this study, the extent of coagulation necrosis was significantly more extensive in the DAM group than that in the SAM group. The extent of coagulation necrosis was measured, using the white area at the center as the border of coagulation necrosis. In this area, all cells were irreversibly damaged, 
and the congestive site at the periphery was excluded(15). Although there was no statistically significant difference in EI between the groups, macroscopically, the shape of coagulation necrosis tended to be non-spherical in the SAM group and spherical in the DAM group. It should be noted that the extent of coagulation necrosis depends on many factors such as RFA conditions including the area of ablation such as the perivascular area, the type of electrode needle used, heat conduction/ convection, low-resistance shunt, and the position/ distance of the contra-polar plate. We previously reported that, when ablation is performed around blood vessels or at a vascular site, blood flow-related cooling effects influence the extent of ablation (10). In this study, the sites for the single/double ablation were randomly selected from areas in which there were no major blood vessels, as evidenced by ultrasonography. Clinically, however, patients with HCCs usually have a cirrhotic liver, and the vasculatures of HCCs are different from non-tumor livers. A cirrhotic liver, as well as liver tumor, might have a different conductivity for the RF current(16). At any rate, a double ablation with a multitined expandable electrode for RFA could also be useful in HCC patients compared to a single ablation.

No marked difference in the interval between the start of the ablation and the first roll-off was found between the single and double ablation groups. In the DAM group, an additional ablation was started at a lower output than that used for the first ablation, and the interval until roll-off was prolonged. Therefore, the difference between the two groups can be attributed to the second ablation. Excessive current output may induce excessive heat, boiling, tissue dryness, and carbonization in the early stages, resulting in an earlier roll-off(18). However, the second ablation at a low output may have affected peripheral tissue to some extent via coagulation necrosis.

In conclusion, we have demonstrated in the present study that the use of the double ablation method for radiofrequency ablation was more extensive with a spherical zone shape compared to a single ablation.

\section{ACKNOWLEDGMENTS}

We wish to thank Boston Scientific Japan, Inc. for its support. The animal experiment was performed at the Miyazaki Technology and Education Center.

\section{REFERENCES}

1. Mulier S, Mulier P, Ni Y, Miao Y, Dupas B, Marchal G, De Wever I, Michel L: Complications of radiofrequency coagulation of liver tumours. Br J Surg 89 : 1206-1222, 2002

2. Tateishi R, Shiina S, Teratani T, Obi S, Sato S, Koike Y, Fujishima T, Yoshida H, Kawabe T, Omata M : Percutaneous radiofrequency ablation for hepatocellular carcinoma. Ananalysis of 1000 cases. Cancer 103 : 1201-1209, 2005

3. Rossi S, Di Stasi M, Buscarini E, Quaretti P, Garbagnati F, Squassante L, Paties CT, Silverman DE, Buscarini L : Percutaneous RF interstitial thermal ablation in the treatment of hepatic cancer. AJR Am J Roentgenol 167 : 759-768, 1996

4. Livraghi T, Goldberg SN, Lazzaroni S, Meloni F, Solbiati L, Gazelle GS : Small hepatocellular carcinoma : treatment with radio-frequency ablation versus ethanol injection. Radiology 210 : 655-661, 1999

5. Curley SA, Izzo F, Ellis LM, Nicolas Vauthey J, Vallone P : Radiofrequency ablation of hepatocellular cancer in 110 patients with cirrhosis. Ann Surg 232 : 381-391, 2000

6. Shiina S, Teratani T, Obi S, Sato S, Tateishi R, Fujishima T, Ishikawa T, Koike Y, Yoshida H, Kawabe T, Omata M : A randomized controlled trial of radiofrequency ablation with ethanol injection for small hepatocellular carcinoma. Gastroenterology 129 : 122-130, 2005

7. Shibata T, Iimuro $Y$, Yamamoto $Y$, Maetani $Y$, Ametani F, Itoh K, Konishi J : Small hepatocellular carcinoma : comparison of radio-frequency ablation and percutaneous microwave coagulation therapy. Radiology $223: 331-337,2002$

8. Curley SA, Marra P, Beaty K, Ellis LM, Vauthey JN, Abdalla EK, Scaife C, Raut C, Wolff R, Choi H, Loyer E, Vallone P, Fiore F, Scordino F, De Rosa V, Orlando R, Pignata S, Daniele B, Izzo F : Early and late complications after radiofrequency ablation of malignant liver tumors in 608 patients. Ann Surg 239 : 450-458, 2004

9. de Baere T, Risse O, Kuoch V, Dromain C, Sengel C, Smayra T, Gamal El Din M, Letoublon C, Elias D : Adverse events during radiofrequency treatment of 582 hepatic tumors. AJR Am J Roentgenol 181 : 695-700, 2003

10. Tamaki K, Shimizu I, Oshio A, Fukuno H, Inoue $\mathrm{H}$, Tsutsui A, Shibata H, Sano N, Ito S : 
Influence of large intrahepatic blood vessels on the gross and histological characteristics of lesions produced by radiofrequency ablation in a pig liver model. Liver Int 24 : 696701, 2004

11. Mulier S, Burdio F, Denys AL, Dupas B, Habib N, Hoey M : Experimental radiofrequency ablation : Proposal for standardized description of size and geometry of the ablation zone. Ann Surg Oncol (in press, 2006)

12. Mulier S, Miao Y, Mulier P, Dupas B, Pereira P, de Baere T, Lencioni R, Leveillee R, Marchal G, Michel L, Ni Y : Electrodes and multiple electrode systems for radiofrequency ablation: a proposal for updated terminology. Eur Radiol 15 : 798-808, 2005

13. Pereira PL, Trubenbach J, Schenk M, Subke J, Kroeber S, Schaefer I, Remy CT, Schmidt D, Brieger J, Claussen CD : Radiofrequency ablation: in vivo comparison of four commercially available devices in pig livers. Radiology 232 : 482-490, 2004

14. Ni Y, Mulier S, Miao Y, Michel L, Marchal G :
A review of the general aspects of radiofrequency ablation. Abdom Imaging $30: 381-400$, 2005

15. Ng KK, Lam CM, Poon RT, Shek TW, Yu WC, To JY, Wo YH, Lau CP, Tang TC, Ho DW, Fan ST: Porcine liver : morphologic characteristics and cell viability at experimental radiofrequency ablation with internally cooled electrodes. Radiology 235 : 478-486, 2005

16. Glaiberman CB, Pilgram TK, Brown DB : Patient factors affecting thermal lesion size with an impedance-based radiofrequency ablation system. J Vasc Interv Radiol 16 : 1341-1348, 2005

17. Solbiati L, Goldberg N, Ierace T, Livraghi T, Meloni F, Dellanoce M, Sironi S, Gazelle GS : Hepatic metastases : percutaneous radio-frequency ablation with cooled tip electrodes. Radiology 205: 367-373, 1997

18. Miao Y, Ni Y, Yu J, Zhang H, Baert A, Marchal $\mathrm{G}$ : An ex vivo study on radiofrequency tissue ablation : increased lesion size by using an "expandable-wet" electrode. Eur Radiol 11: 1841-1847, 2001 